\title{
Suppression of Vasoactive Intestinal Polypeptide in the Suprachiasmatic Nucleus Leads to Aging-Like Alterations in cAMP Rhythms and Activation of Gonadotropin-Releasing Hormone Neurons
}

\author{
Lynnette M. Gerhold, ${ }^{1}$ Katherine L. Rosewell, ${ }^{2}$ and Phyllis M. Wise ${ }^{1}$ \\ ${ }^{1}$ Department of Neurobiology, Physiology, and Behavior, Division of Biological Sciences, University of California, Davis, Davis, California 95616, and \\ ${ }^{2}$ Department of Obstetrics and Gynecology, College of Medicine, University of Kentucky, Lexington, Kentucky 40536
}

\begin{abstract}
Input from the suprachiasmatic nucleus $(\mathrm{SCN})$ to gonadotropin-releasing hormone $(\mathrm{GnRH})$ neurons is critical to the occurrence of regular cyclic GnRH secretion. It is thought that an essential neuropeptide in the SCN that communicates this cyclic information to GnRH neurons is vasoactive intestinal polypeptide (VIP) and that it may act through cAMP. We tested the hypothesis that (1) aging involves a blunting of cAMP diurnal rhythmicity in the SCN; (2) administration of antisense oligonucleotides (anti-oligos) against VIP, which produces an aging-like pattern in VIP, would lead to an aging-like suppression of cAMP; and (3) this in turn would lead to inhibition of the steroid-induced activation of GnRH neurons. We measured cAMP concentrations in the SCN and rostral preoptic nucleus throughout the day in young and middle-aged rats that were ovariectomized (OVX) or OVX and treated with estradiol.

Our results show that cAMP concentrations exhibit a diurnal rhythm in young rats, and that this rhythm is totally abolished by the time rats are middle age. Administration of antisense oligonucleotides against VIP or random oligos suppresses VIP concentrations and abolishes the cAMP rhythm, leading to significantly reduced activation of GnRH neurons. Together, these findings strongly suggest that the SCN conveys diurnal information to GnRH neurons by driving VIP-dependent cAMP rhythms. In addition, aging involves deterioration in this VIP-driven rhythmicity, which impacts the ability of steroids to induce GnRH neuronal activation.
\end{abstract}

Key words: GnRH; aging; medial preoptic area; vasoactive intestinal polypeptide; cAMP; reproduction

\section{Introduction}

An intricate and complex interplay of hypothalamic and peripheral endocrine events controls the cyclic synthesis and secretion of gonadotropin-releasing hormone $(\mathrm{GnRH})$, which in turn, leads to cyclic secretion of luteinizing hormone ( $\mathrm{LH})$ from the anterior pituitary. The synchronization of these events is tightly coupled to environmental cues, such as the light/dark cycle, and circulating ovarian hormones, such as estradiol (Wise, 1999). As rats approach middle age, age-related deterioration in hypothalamic functions is a crucial element that contributes to reproductive decline (Wise et al., 1997, 1999; Wise, 1999, 2000; Wise and Smith, 2001). We found that the ability of the suprachiasmatic nucleus (SCN), the circadian pacemaker of the brain, to drive an array of diurnal neurochemical events diminishes with age, and this may explain the dampening and desynchronization of multiple neurotransmitter rhythms that lead to the age-related improper timing of GnRH-LH surges (Lloyd et al., 1994; Krajnak et al., 2001; Le et al., 2001). Assessment of activation of GnRH neu-

Received Sept. 1, 2004; revised 0ct. 20, 2004; accepted Nov. 17, 2004.

This work was supported by National Institutes of Health Grants AG02224 and AG17164 (P.M.W.).

Correspondence should be addressed to Dr. Phyllis M. Wise, Division of Biological Sciences, University of California

Davis, One Shields Avenue, Davis, CA 95616-8536. E-mail: pmwise@ucdavis.edu.

DOI:10.1523/JNEUROSCI.3598-04.2005

Copyright $\odot 2005$ Society for Neuroscience $\quad$ 0270-6474/05/250062-06\$15.00/0 rons, by measuring Fos within the nuclei of GnRH neurons (Lee et al., 1990), reveals that although the concentration of $\mathrm{GnRH}$ does not change, the extent of activation is markedly attenuated during middle age (Lloyd et al., 1994; Rubin et al., 1997; Krajnak et al., 2001; Legan and Tsai, 2003). Therefore, decreased activity of GnRH neurons on proestrus and a decrease in the magnitude and a delay in the timing of the preovulatory LH surge characterize this functional decline in reproduction.

Several lines of evidence suggest that a diurnal pattern of vasoactive intestinal polypeptide (VIP) gene and protein expression, originating from the SCN, is an essential component of the constellation of stimulatory signals leading to the proper timing of preovulatory GnRH (Harney et al., 1996; Horvath et al., 1998; van der Beek et al., 1999) and that changes in the dynamics of this neuropeptide may contribute to age-related alterations in the pattern of GnRH secretion (Krajnak et al., 2001; Le et al., 2001). First, VIP neurons in the SCN directly innervate GnRH neurons in the rostral preoptic nucleus (rMPN) (van der Beek et al., 1997; Horvath et al., 1998). Second, GnRH neurons express the VIP/ PACAP receptor subtype $2\left(\mathrm{VPAC}_{2}\right)$ receptor subtype (Smith et al., 2000), which is coupled to the stimulatory G-protein (Gs) signal transduction pathway that leads to an accumulation of cAMP (McCulloch et al., 2002). Interestingly, Chappell et al. (2000) reported that cAMP levels in the anteroventral periven- 
tricular nucleus (AVPV) exhibit a diurnal rhythm that is markedly analogous to the rhythm of VIP. Third, aging affects the rhythm of VIP in the SCN (Krajnak et al., 1998a), and suppression of this rhythm in young rats leads to delayed and attenuated LH surges that are strikingly similar to those observed in middleaged (MA) female rats (Harney et al., 1996; van der Beek et al., 1999).

The goal of this study was to determine whether the diurnal rhythm of cAMP in the SCN and rMPN changes with age, and whether attenuation of VIP leads to a disruption of the cAMP diurnal rhythm and concomitant suppression of steroid-induced activation of GnRH neurons.

\section{Materials and Methods}

Animals. Young (2-3 months of age) and middle-aged (9-12 months of age) female Sprague Dawley rats (Zivic-Miller, Penelope, PA) were maintained on a 14/10 hr light/dark cycle (lights on at $0400 \mathrm{~h}$ ) with food and water available ad libitum. Estrous cyclicity was monitored by daily vaginal lavage for at least 3 weeks before use. Only young rats that exhibited at least two consecutive $4 \mathrm{~d}$ estrous cycles and middle-aged rats that exhibited estrous cycles of regular or irregular length (5-8 d) were used. All rats were ovariectomized (OVX; day 0 ) and implanted subcutaneously with a SILASTIC capsule ( $0900 \mathrm{~h}$ on day 7; Konigsberg Instruments, Pasadena CA) containing either sesame oil or $17 \beta$ estradiol (Sigma, St. Louis, MO) dissolved in sesame oil ( $180 \mu \mathrm{g} / \mathrm{ml}$; young, 30 $\mathrm{mm}$ capsule; middle-aged, $40 \mathrm{~mm}$ capsule; $0.062 \times 0.125$ inches, inner $\times$ outer diameter). This estradiol treatment paradigm produces estradiol levels $\sim 15 \mathrm{pg} / \mathrm{ml}$ by day 2 after implantation (Wise et al., 1981). Animals were killed $2 \mathrm{~d}$ later (day 9) at 0300, 0800, 1200, 1400, 1600, and $1800 \mathrm{~h}$ ( $n=8 \mathrm{rats} /$ time point/treatment/age). Brains were collected, rapidly frozen, and stored at $-80^{\circ} \mathrm{C}$ until sectioned. Frozen coronal sections $(300 \mu \mathrm{m})$ through the hypothalamus were mounted on glass slides. Using modified stainless steel needles, the rMPN (17 gauge), SCN (21 gauge), and cortex (17 gauge) were microdissected and stored at $-80^{\circ} \mathrm{C}$ until processed. The organun vasculosum of the lamina terminalisrMPN contains the subpopulation of GnRH neurons that regulate $\mathrm{LH}$ secretion (Swanson, 1987; Petersen et al., 1995). We chose to examine these areas based on previous work showing that VIP predominately innervates the GnRH neurons in this region and that GnRH neurons that are lateral to these regions were not included (van der Beek et al., 1997; Krajnak et al., 2001)

Stereotaxic surgery. Stereotaxic surgeries were performed based on the protocols of Harney et al. (1996) and Gerhold et al. (2002), which are described below. Five days after OVX (day 5), rats were anesthetized with ketamine $(49 \mathrm{mg} / \mathrm{ml})-x y l a z i n e ~(1.8 \mathrm{mg} / \mathrm{ml})$ and implanted with bilateral guide tubes directed stereotaxically at the SCN (1.5 mm apart; $9 \mathrm{~mm}$ in length; 26 gauge; Plastics One, Roanoke, VA). Two days after bilateral guide tube implantation (day 7), VIP antisense or random sequence oligos ( $0.5 \mu \mathrm{g}$ in $0.5 \mu \mathrm{l}$ of saline per side; Invitrogen, Carlsbad, CA) were infused over a $4 \mathrm{~min}$ interval at $0800 \mathrm{~h}$ through cannulas implanted bilaterally in the guide tubes. The antisense oligonucleotides were 20 nucleotides in length complementary to the cap site (5'-GCTCTGCACTACAACCTGAC- $3^{\prime}$ ) and translation start site (5'-TTGCTTCTGGATTCCATCTC-3') of the rat VIP mRNA (Giladi et al., 1990). Control oligonucleotides had the same ATGC content as antisense oligonucleotides but in random order that had no significant homology to any known peptide localized in the SCN. At the same time, rats were implanted with SILASTIC capsules containing estradiol dissolved in sesame oil or sesame oil alone $(180 \mu \mathrm{g} / \mathrm{ml} ; 30 \mathrm{~mm}$ capsule). Rats were killed $2 \mathrm{~d}$ later (day 9) at $43 \mathrm{hr}(0300 \mathrm{~h}), 53 \mathrm{hr}(1400 \mathrm{~h})$, and $57 \mathrm{hr}(1800 \mathrm{~h})$ after injection. Using this endocrine paradigm, $\mathrm{LH}$ surges occur between 1300 and $1900 \mathrm{~h}$, with peak concentrations at $\sim 1500-1600 \mathrm{~h}$ (Wise et al., 1981). In one group, the rMPN, one side of the SCN, and cortex were microdissected as described above and prepared for ELISA to measure cAMP levels. In the second group, antisense and random sequence oligotreated rats as well as a group of middle-aged regularly cycling female rats were transcardially perfused with $4 \%$ paraformaldehyde (Sigma), and the brains were removed, sectioned coronally, and stored in cryoprotectant at $-20^{\circ} \mathrm{C}$ until processed for immunocytochemistry (ICC). The SCN was checked for correct cannulas placement as the brains were sectioned ( $n=6-10 \mathrm{rats} /$ time point/treatment).

cAMP and protein analysis. Tissue samples were homogenized with a Sonic Dismembrator (Fisher Scientific, Houston, TX) in ice-cold 5\% trichloroacetic acid (Sigma). Homogenates were centrifuged at $600 \times g$, and supernatants were removed, placed in microcentrifuge tubes, lyophilized in a Speed-Vac evaporator (Savant Instruments, Farmington, NY), and reconstituted in assay buffer and measured for cAMP (Assay Designs, Ann Arbor, MI). Pellets were resuspended in buffer solution from the cAMP kit. Protein content (10 $\mu$ l of reconstituted homogenates) was determined using the Bradford assay (Bio-Rad, Hercules, CA). Data were expressed as picomoles of cAMP per milligram of protein.

Immunocytochemistry. Coronal brain sections $(40 \mu \mathrm{m})$ were cut in a cryostat (Microm, Kalamazoo, MI) starting at the level of the medial diagonal band of Broca (bregma, $0.20 \mathrm{~mm}$ ) (Paxinos and Watson, 1997) through the arcuate nucleus (bregma, $-3.80 \mathrm{~mm}$ ). Each brain was collected in a series of six sets with every sixth section represented in a group and stored in cryoprotectant until processed for ICC. Antibodies against GnRH (LR-1; Benoit, Montreal, Canada) and Fos (Santa Cruz Biotechnology, Santa Cruz, CA) (recognizes Fos and Fra-related antigen) were used to determine activation of GnRH neurons. On day 1, sections from one series that contained every sixth section were rinsed in $0.1 \mathrm{M}$ Trisbuffered saline (TBS) and blocked with 10\% normal horse serum (NHS) plus $0.4 \%$ Triton X-100 (NHS-X) for $1 \mathrm{hr}$ at room temperature. Next, sections were incubated in goat anti-Fos $(1: 25,000)$ in $2 \%$ NHS-X overnight at $4^{\circ} \mathrm{C}$. On day 2 , sections were rinsed in TBS, incubated in biotinylated anti-goat IgG diluted 1:500 in 2\% NHS-X for $1 \mathrm{hr}$ at room temperature, followed by incubation in avidin-biotin complex (ABC) (Vectastain kit; Vector Laboratories, Burlingame, CA) in TBS plus $0.4 \%$ Triton X-100 for $1 \mathrm{hr}$ at room temperature. Finally, sections were incubated in $0.025 \%$ diaminobenzidine (DAB; Sigma) with $0.02 \%$ ammonium nickel sulfate (Fisher Scientific) and $0.1 \mu \mathrm{l} / \mathrm{ml}$ of $30 \%$ hydrogen peroxide in TBS for $10 \mathrm{~min}$ at room temperature. After the DAB reaction, sections were rinsed in TBS and then incubated in rabbit anti-GnRH $(1: 100,000)$ in $2 \%$ NHS-X overnight at $4^{\circ} \mathrm{C}$. On day 3 , sections were rinsed in TBS, incubated in biotinylated anti-rabbit IgG diluted 1:500 in $2 \%$ NHS-X for $1 \mathrm{hr}$ at room temperature, followed by incubation in $\mathrm{ABC}$ (Vectastain kit; Vector Laboratories) in TBS plus $0.4 \%$ Triton X-100 for $1 \mathrm{hr}$ at room temperature. Finally, sections were incubated in $0.025 \%$ DAB (Sigma) with $0.1 \mu \mathrm{l} / \mathrm{ml}$ of $30 \%$ hydrogen peroxide in TBS for 10 $\mathrm{min}$ at room temperature. Each step was followed by rinses in TBS [three rinses in TBS (5 min each)]. Sections were mounted on slides, and coverslips were applied using Permount (Fisher Scientific).

VIP radioimmunoassay total protein in SCN. In antisense-treated animals, the frozen SCN tissue punches were divided into two groups for each animal. One group was used in the cAMP ELISA. The other group was used to determine the concentration of VIP in the SCN tissue punches. Tissue punches were extracted as described above. The pellets were assayed for VIP using a kit (Peninsula Laboratories, Belmont, CA). Total protein in the SCN punches was measured using the DC Protein Assay kit (Bio-Rad). Data were expressed as picograms of VIP per microgram of protein.

Statistical analysis. cAMP levels in the rMPN, SCN, and cerebral cortex were analyzed by Student's $t$ test. VIP concentrations in the SCN were measured by two-way ANOVA followed by Bonferroni's post hoc test for effects of treatment $\times$ time interaction. One-way ANOVA followed by Newman-Keuls post hoc tests were performed on cAMP concentrations in antisense and random oligo-treated animals in the rMPN, SCN, and cerebral cortex to test for effects of time. Two-way ANOVA followed by Bonferroni's post hoc test were used to determine the percentage of GnRH expressing compared with all GnRH neurons in antisense and random sequence oligo-treated rats for effects, treatment, time, and treatment $\times$ time interactions. All statistics were performed using Prism 4.0 software (Graphpad, San Diego, CA). 
Results

cAMP

SCN

In young OVX and OVX rats treated with estradiol (OVX-E ${ }_{2}$ ), cAMP levels (picomoles per milligram of protein) in the SCN exhibited a diurnal rhythm (Fig. $1 A$ ). Regardless of steroidal milieu, cAMP levels were the lowest at 0300 and $0800 \mathrm{~h}$ (Fig. $1 A)$. By $1200 \mathrm{~h}$, cAMP levels rose and remained high through 1400 h. By 1600 h, cAMP levels returned to baseline and were not significantly different from 0300 and 0800 h. In middle-aged OVX and OVX$\mathrm{E}_{2}$-treated rats, cAMP levels did not exhibit a diurnal rhythm (Fig. $1 B$ ).

\section{$r M P N$}

In young rats, cAMP levels (picomoles per milligram of protein) exhibited diurnal rhythms that were similar regardless of steroidal milieu (Fig. 1C). In OVX rats, cAMP levels were the lowest at 0300 and $0800 \mathrm{~h}$ (Fig. 1C). By 1200 h, cAMP levels rose and peaked at 1400 and $1600 \mathrm{~h}$. By $1800 \mathrm{~h}$, cAMP levels returned to baseline and were not significantly different from 0300 and $0800 \mathrm{~h}$. In OVX-E $\mathrm{E}_{2}$-treated young rats (Fig. 1C), cAMP levels were low at $0300 \mathrm{~h}$, $0800 \mathrm{~h}$, and $1200 \mathrm{~h}$, peaked at 1400 and $1600 \mathrm{~h}(p<0.05)$, and returned to baseline at $0600 \mathrm{~h}$. In middle-aged rats, cAMP levels did not exhibit a diurnal rhythm in either

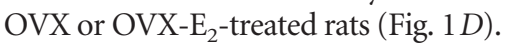

\section{Cerebral cortex}

cAMP (picomoles per milligram of protein) did not exhibit a diurnal rhythm in the cortex, and neither $\mathrm{E}_{2}$ nor age altered the pattern or level of cAMP (Fig. $1 E, F)$.
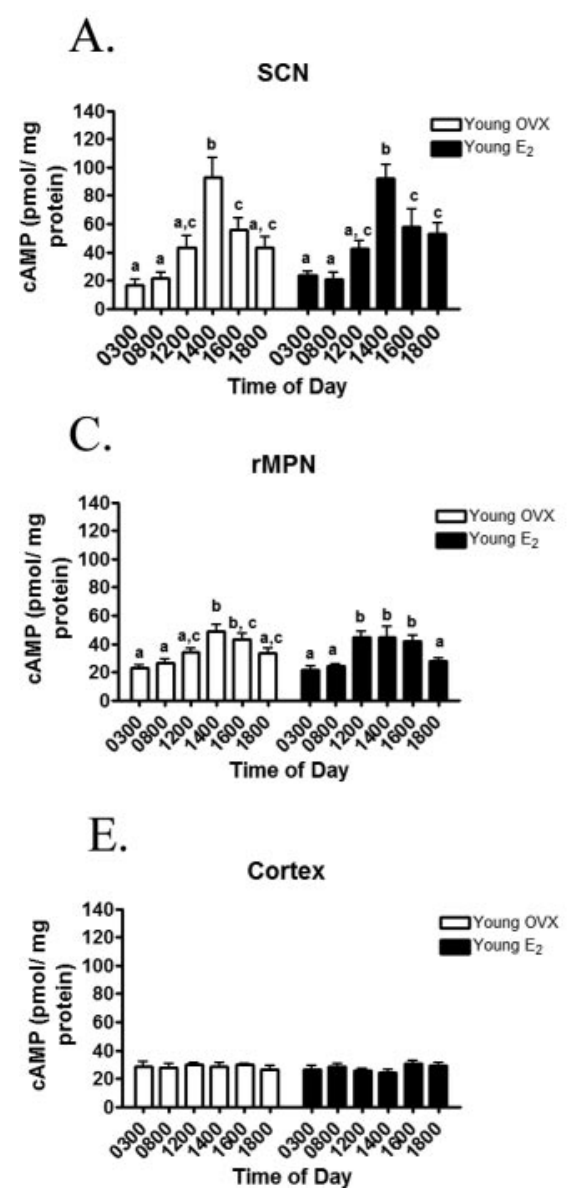

B.

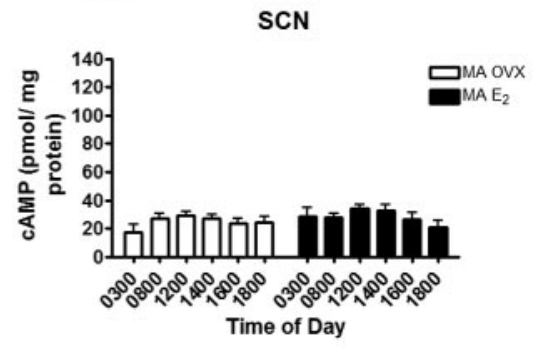

D.



F.



Figure 1. A, CAMP levels in the $\mathrm{SCN}$ of young rats exhibit a diurnal rhythm, and treatment with estradiol $\left(\mathrm{E}_{2}\right)$ does not affect this rhythm. $B$, cAMP levels in the $S C N$ of MA rats do not exhibit a diurnal rhythm, regardless of treatment with $E_{2}$. C, cAMP levels (picomoles per milligram of protein) in the rMPN of young rats exhibit a diurnal rhythm, and treatment with estradiol $\left(E_{2}\right)$ does not affect this rhythm. D, cAMP levels in the rMPN of MA rats do not exhibit a diurnal rhythm, regardless of treatment with $E_{2} . E_{1} F$, cAMP levels in the cortex do not exhibit diurnal rhythms, regardless of treatment or age. Bars with dissimilar letters represent significance of at least $p<0.05$.

\section{Effects of antisense oligo treatment on VIP concentrations in the SCN}

VIP levels (picograms per microgram of protein) in the SCN in random sequence oligo-treated rats displayed a diurnal rhythm (Fig. 2). VIP concentrations were low at $0300 \mathrm{~h}$ (43 hr after injection), significantly greater at $1400 \mathrm{~h}$ ( $53 \mathrm{hr}$ after injection; $p<0.05)$, and decreased by $1800 \mathrm{~h}$ ( $57 \mathrm{hr}$ after injection). Treatment with antisense oligo against VIP blocked the VIP rhythm (Fig. 2).

Effects of antisense oligo treatment on cAMP concentrations in the SCN, rMPN, and cerebral cortex

cAMP levels in the SCN and rMPN of control random sequence oligo-treated rats displayed a diurnal rhythm similar to cAMP levels in Figure 1 (Fig. $3 A$ ). In the SCN, cAMP levels in control random sequence oligo-treated rats were lower at $0300 \mathrm{~h}$ (Fig. $3 A)$ and increased at $1400 \mathrm{~h}(p<0.001)$. By $1800 \mathrm{~h}$, cAMP levels were significantly decreased compared with $1400 \mathrm{~h}(p<0.001)$ but were still more than at $0300 \mathrm{~h}(p<0.05)$. Antisense oligo treatment suppressed cAMP levels and abolished the rhythm (Fig. $3 A$ ). In the rMPN, cAMP levels were significantly low at $0300 \mathrm{~h}$ and increased at $1400 \mathrm{~h}(p<0.001)$ and returned to baseline (Fig. 3B). Antisense oligo treatment significantly

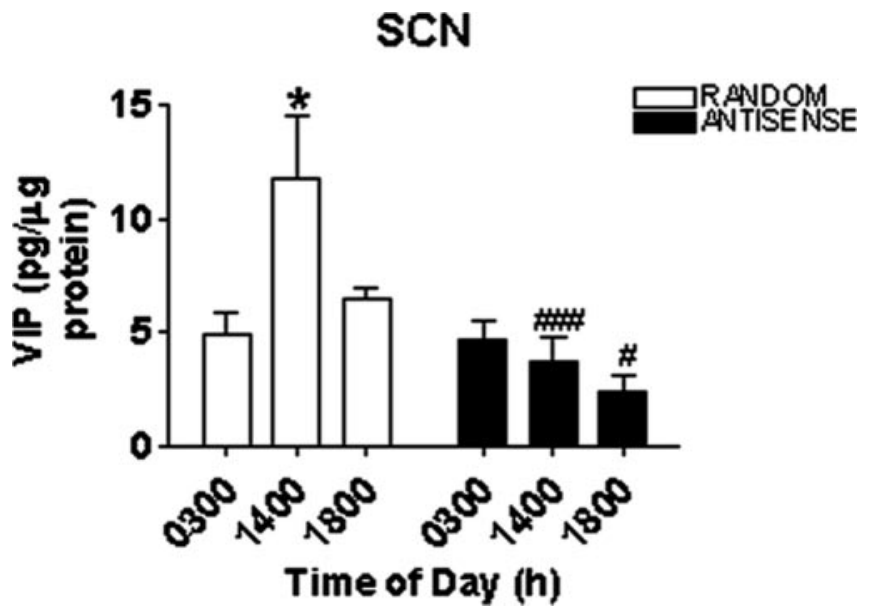

Figure 2. VIP levels (picograms per microgram of protein) in the $\mathrm{SCN}$ of rats treated with random sequence oligos or antisense oligos. VIP exhibits a diurnal rhythm in rats treated with random sequence oligos, whereas treatment with antisense oligos disrupts the VIP diurnal rhythm. Symbols indicate levels of significance between time points ( $\left.{ }^{*} p<0.05\right)$ and between treatments $\left(\# p<0.05 ;{ }^{\# \#} p<0.001\right)$. 

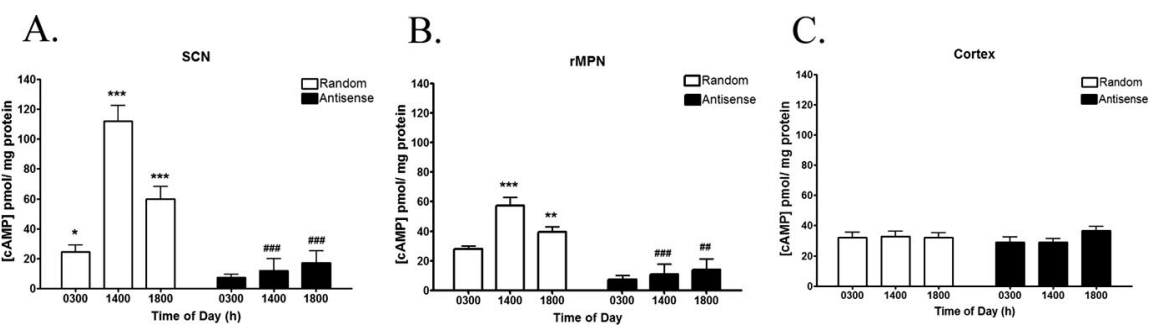

Figure 3. A, CAMP levels (picomoles per milligram of protein) in the $S C N$ of rats treated with random sequence oligos exhibit a diurnal rhythm, whereas treatment with antisense oligos disrupts the CAMP diurnal rhythm. $B$, cAMP levels (picomoles per milligram of protein) in the rMPN of rats treated with random sequence oligos exhibit a diurnal rhythm, whereas treatment with antisense oligos disrupts the CAMP diurnal rhythm. C, cAMP levels in the cortex do not exhibit diurnal rhythms, regardless of treatment. Symbols indicate levels of significance between time points $\left({ }^{*} p<0.05 ;{ }^{* *} p<0.01 ;{ }^{* * *} p<0.001\right)$ and between treatments $\left({ }^{\# \#} p<0.01 ;{ }^{\# \# \#} p<0.001\right)$.
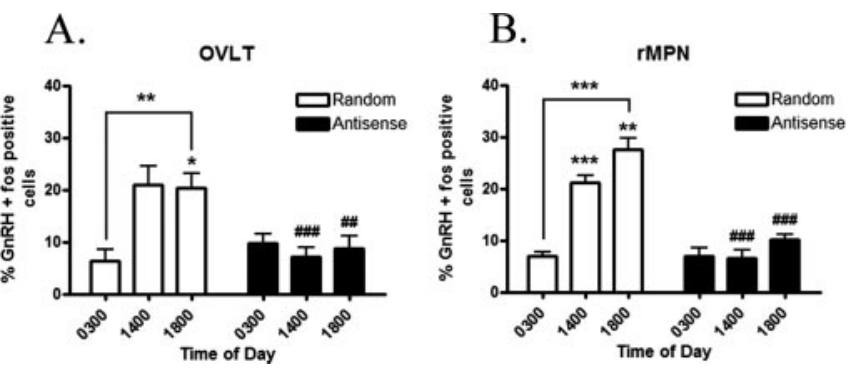

Figure 4. The percentage of GnRH neurons expressing Fos in the $\operatorname{OVLT}(A)$ and the $\operatorname{rMPN}(B)$ of rats treated with random sequence oligos throughout the day exhibited a diurnal rhythm. Treatment with antisense oligos in the SCN disrupted the diurnal rhythm in the OVLT and rMPN. Symbols indicate levels of significance between time points $\left({ }^{*} p<0.05\right.$; ${ }^{* *} p<0.01$; ${ }^{* *} p<$ $0.001)$ and between treatments $\left({ }^{\# \#} p<0.01 ;{ }^{\# \# \#} p<0.001\right)$.
A.



B.



Figure 5. GnRH neurons expressing Fos in the $\operatorname{OVLT}(A)$ and in the $\operatorname{rMPN}(B)$ in middle-aged rats did not exhibit a diurnal rhythm and were not different from the antisense oligo-treated rats.

decreased cAMP levels in the rMPN at all time points and abolished the rhythm (Fig. 3B). Antisense treatment did not significantly affect cAMP levels in the cerebral cortex (Fig. 3C).

\section{Effects of antisense oligo treatment on $\mathrm{GnRH}$ activation in the} OVLT and rMPN compared with MA rats

The percentage of GnRH expressing Fos immunoreactivity (IR) in the OVLT (Fig. 4A) and rMPN (Fig. 4B) in control random sequence oligo-treated rats was low at $0300 \mathrm{~h}$ and rose at $1400 \mathrm{~h}$ $(p<0.01)$ and $1800 \mathrm{~h}(p<0.001)$. In previous studies, we have established that the LH surge occurs between 1400 and $1800 \mathrm{~h}$. Thus, the expression of Fos within GnRH neurons is an index of activation of these neurons and stimulation of the LH surge. In rats treated with antisense oligos to VIP, the percentage of GnRH neurons expressing Fos-IR in the OVLT and rMPN did not rise at 1400 or $1800 \mathrm{~h}(p<0.001)$ (Fig. $4 A, B)$. We were interested to determine whether antisense treatment mimicked the level of $\mathrm{GnRH}$ activation in middle-aged rats. Therefore, in parallel, we analyzed a group of estradiol-treated middle-aged rats. The percentage of GnRH-expressing Fos-IR was identical to antisense-treated rats and did not show the characteristic afternoon activation that we observed in young rats in either the OVLT (Fig. 5A) or the rMPN (Fig. 5B).

\section{Discussion}

Our study clearly establishes four important points. First, in young rats, cAMP levels exhibit a robust diurnal rhythm in the SCN and rMPN, two brain regions that play crucial roles in the occurrence of cyclic GnRH release. Second, this rhythm in both brain regions disappears by the time rats are middle-aged, a time when cyclic GnRH release and reproductive cycles become irregular. Third, VIP from the SCN appears to drive this rhythm, because suppression of the VIP rhythm abolishes the cAMP rhythm in both brain regions. Fourth, the VIP-driven cAMP rhythm plays a critical role in activating GnRH neurons, because blockade of the VIP rhythm suppresses this activation. Thus, we have established, for the first time, a functional relationship between VIP, cAMP, and GnRH neurons, a pathway that appears to be important in reproductive cyclicity.

The SCN of the hypothalamus is the major circadian pacemaker of the brain and consists of the following: (1) biological oscillators that are endogenous and maintain a self-sustained circadian periodicity in the absence of environmental time cues; (2) input pathways that convey environmental information, especially light cues, that entrain circadian oscillations to local time; and (3) output pathways that drive overt circadian rhythms, such as the rhythms of locomotor activity and a variety of endocrine rhythms (Goldman, 1999). Ablation of the SCN disrupts virtually all rhythms and leads to the disappearance of cyclic reproductive function in female rodents (Bethea and Neill, 1980).

Our results clearly establish that cAMP rhythms exist in the SCN and $\mathrm{rMPN}$, and that aging involves a waning of these cAMP rhythms in middle-aged rats. These brain regions work in concert to regulate the cyclic synthesis and secretion of $\mathrm{GnRH}$ and to induce reproductive behaviors (Wise and Smith, 2001). Our findings confirm and extend the previous study by Chappell et al. (2000) who showed that cAMP levels display a diurnal rhythm in the AVPV. It is particularly intriguing that the disappearance of the cAMP rhythms occur at the same age as previously reported alterations in VIP rhythms in the SCN and in the activation of $\mathrm{GnRH}$ neurons, which is required for LH surges to occur (Lloyd et al., 1994; Krajnak et al., 1998a, 2001; Le et al., 2001; Legan and Tsai, 2003). Therefore, we explored whether these three neurochemicals (VIP, cAMP, and GnRH) are functionally linked.

We asked whether VIP drives the cAMP rhythm. VIP is a logical candidate for several reasons. First, VIP is a major neuropeptide in the $\mathrm{SCN}$ and receives retinal projections directly from the retinohypothalamic tract (Stephan et al., 1981; Tanaka et al., 1993) and indirectly from the retinorecipient intergeniculate leaflet via the geniculohypothalamic tract (Tanaka et al., 1993). Second, VIP mRNA and protein exhibit diurnal rhythmicity and are entrained to the light/dark cycle (Shinohara et al., 1993; Krajnak et al., 1998b). Third, VIP binding to the VPAC 2 receptor activates the Gs pathway leading to the accumulation of cAMP (McCulloch et al., 2002). Fourth, we have shown that the rhythm in VIP mRNA in the SCN disappears during middle age 
(Krajnak et al., 1998a), at the same age we observed the disappearance in cAMP rhythms in the current study. VIP dynamics are sexually dimorphic and play essential roles in cyclic reproductive function in females. The number of VIP boutons that terminate on GnRH neurons and the percentage of GnRH neurons contacted by VIP axons are greater in female rats than in male rats (Horvath et al., 1998). Krajnak et al. (1998b) showed that the rhythm of VIP mRNA in the SCN of females is $12 \mathrm{hr}$ out of phase with that of male rats. In females, peak VIP mRNA occurs during the early afternoon of the light phase, whereas in males, peak VIP mRNA and protein occur in the middle of the dark phase. This sexually dimorphic pattern of VIP activity gave rise to the speculation that VIP neurons are critical in conveying circadian information to GnRH neurons and may indicate the involvement of VIP in the sex-specific regulation of GnRH release in rats.

To test whether VIP drives the cAMP rhythm, we administered antisense oligos to VIP into the peri-SCN region of young rats. This treatment had three effects: (1) it suppressed the concentrations of VIP in the SCN, replicating the effects that we observed previously (Harney et al., 1996); (2) it abolished the rhythm of VIP peptide expression; thus, both the concentration and absence of rhythmicity mimicked the profile normally observed in MA rats (Krajnak et al., 1998a); and (3) it suppressed cAMP rhythms in the SCN and rMPN, a profile that strikingly resembles the MA rats. Thus, we conclude that VIP may be the stimulus of the cAMP rhythms and that inducing an aging like profile in VIP leads to an equivalent aging-like profile in cAMP.

Finally, we tested whether the VIP-driven cAMP rhythm is functionally related to the activation of GnRH neurons. We tested this hypothesis based on several lines of experimental evidence: (1) VIP fibers from the SCN innervate GnRH neurons (van der Beek et al., 1997; Horvath et al., 1998); (2) GnRH neurons express the $\mathrm{VPAC}_{2}$ receptors (Smith et al., 2000); and (3) $\mathrm{GnRH}$ neurons that are closely apposed to VIP fibers preferentially express Fos, an index of activity (Krajnak et al., 2001). We show that injections of VIP antisense oligos into the SCN decreased the level of estradiol-induced Fos activation in $\mathrm{GnRH}$ neurons in the OVLT and rMPN in the afternoon at a time when we observed elevated GnRH activation in young rats. This antisense-induced suppression of $\mathrm{GnRH}$ neuronal activation is similar to what we observed in middle-aged rats and suggests that although VIP innervation of $\mathrm{GnRH}$ neurons does not change with age (Krajnak et al., 2001), the SCN itself may not be functioning properly to activate GnRH neurons.

Our results clearly demonstrate that an aging-like pattern of VIP in the SCN leads to an aging-like profile in cAMP rhythms in the SCN and may lead to an aging-like profile in cAMP rhythms in the rMPN, which in turn may lead to an aging-like suppression of GnRH neuronal activation. Our findings add to the increasing body of evidence that aging results in fundamental changes in the SCN because of the following: (1) the size and number of different neuronal populations change with age (Roozendaal et al., 1987; Chee et al., 1988); (2) the amplitude of electrical activity rhythms in slice and dispersed cultures becomes dampened (Satinoff et al., 1993; Ruby et al., 1998; Aujard et al., 2001); and (3) the free-running period and the light-induced production of the clock genes Per 1 and Per 2 change with age (Yamazaki et al., 2002; Asai et al., 2001). These changes in the pacemaker itself or the coupling to its outputs may cause temporal desynchronization of rhythms that are critical for the precise timing of $\mathrm{GnRH}$ secretion and ultimately LH secretion (Wise et al., 1996, 1997; Krajnak et al., 1998a).

The AVPV, OVLT, and rMPN play critical and interactive roles regulating cyclic GnRH-LH surges. The AVPV serves as a nodal relay station where information on steroidal milieu is communicated to GnRH neurons (Simerly et al., 1990; Simerly, 1998; Simonian et al., 1999). We show that the SCN sends circadian signals directly to the OVLT and rMPN areas of the brain that contain a subpopulation of $\mathrm{GnRH}$ neurons that are dedicated to regulating the cyclic timing of $\mathrm{GnRH}-\mathrm{LH}$ surges. Both the steroidal and circadian information converging on GnRH neurons are necessary but not sufficient by themselves to induce GnRH-LH surges. We demonstrate how age leads to deterioration in the circadian signal that is relayed to $\mathrm{GnRH}$ neurons and how this circadian signal is important in the timing of the GnRH-LH surges. Our microdissected punches contained the AVPV, rMPN, and preoptic area (POA). Thus, it is possible that inputs from the POA could affect GnRH activation. However, because we reduced cAMP and the level of activation of GnRH neurons with the VIP antisense oligo treatment, we conclude that VIP from the SCN is necessary for the timing of the LH surge but not sufficient to induce a LH surge.

In summary, we have established a functional relationship between VIP in the SCN and GnRH neurons in the rMPN that is mediated through the cAMP pathway. These data suggest that VIP is a key messenger from the SCN that communicates timeof-day information to GnRH neurons. Thus, maintenance of the normal rhythm of VIP is of great importance to the synchronization of the SCN with GnRH neurons. Additionally, we have uncovered that aging affects this pathway and may be one component that contributes to the gradual deterioration of reproductive cyclicity in aging females. Subtle changes in the ability of the biological clock to drive reproductive rhythms in middle-aged rats may underlie the transition to acyclicity and decline in reproductive function. Thus, these findings demonstrate the crucial role the SCN plays in coordinating the timing of GnRH-LH surges and reproduction.

\section{References}

Asai M, Yoshinobu Y, Kaneko S, Mori A, Nikaido T, Moriya T, Akiyama M, Shibata S (2001) Circadian profile of Per gene mRNA expression in the suprachiasmatic nucleus, paraventricular nucleus, and pineal body of aged rats. J Neurosci Res 66:1133-1139.

Aujard F, Herzog ED, Block GD (2001) Circadian rhythms in firing rate of individual suprachiasmatic nucleus neurons from adult and middle-aged mice. Neuroscience 106:255-261.

Bethea CL, Neill JD (1980) Lesions of the suprachiasmatic nuclei abolish the cervically stimulated prolactin surges in the rat. Endocrinology 107:1-5.

Chappell PE, Lee J, Levine JE (2000) Stimulation of gonadotropin-releasing hormone surges by estrogen. I. Role of hypothalamic progesterone receptors. Endocrinology 141:1477-1485.

Chee CA, Roozendaal B, Swaab DF, Goudsmit E, Mirmiran M (1988) Vasoactive intestinal polypeptide neuron changes in the senile rat suprachiasmatic nucleus. Neurobiol Aging 9:307-312.

Gerhold LM, Sellix MT, Freeman ME (2002) Antagonism of vasoactive intestinal peptide mRNA in the suprachiasmatic nucleus disrupts the rhythm of FRAs expression in neuroendocrine dopaminergic neurons. J Comp Neurol 450:135-143.

Giladi E, Shani Y, Gozes I (1990) The complete structure of the rat VIP gene. Mol Brain Res 7:261-267.

Goldman BD (1999) The circadian timing system and reproduction in mammals. Steroids 64:679-685.

Harney JP, Scarborough K, Rosewell KL, Wise PM (1996) In vivo antisense antagonism of vasoactive intestinal peptide in the suprachiasmatic nuclei causes aging-like changes in the estradiol-induced luteinizing hormone and prolactin surges. Endocrinology 137:3696-4701.

Horvath TL, Cela V, van der Beek EM (1998) Gender-specific apposition between vasoactive intestinal peptide-containing axons and gonadotrophinreleasing hormone-producing neurons in the rat. Brain Res 795:277-281.

Krajnak K, Kashon ML, Rosewell KL, Wise PM (1998a) Aging alters the 
rhythmic expression of vasoactive intestinal polypeptide mRNA but not arginine vasopressin mRNA in the suprachiasmatic nuclei of female rats. J Neurosci 18:4767-4774.

Krajnak K, Kashon ML, Rosewell KL, Wise PM (1998b) Sex differences in the daily rhythm of vasoactive intestinal polypeptide but not arginine vasopressin messenger ribonucleic acid in the suprachiasmatic nuclei. Endocrinology 139:4189-4196.

Krajnak KM, Rosewell KL, Wise PM (2001) Fos-induction in gonadotropinreleasing hormone neurons receiving vasoactive intestinal polypeptide innervation is reduced in middle-aged female rats. Biol Reprod 64:1160-1164.

Le WW, Wise PM, Murphy AZ, Coolen LM, Hoffman GE (2001) Parallel declines in Fos activation of the medial anteroventral periventricular nucleus and LHRH neurons in middle-aged rats. Endocrinology 142:4976-4982.

Lee WS, Smith MS, Hoffman GE (1990) Luteinizing hormone-releasing hormone neurons express Fos protein during the proestrous surge of luteinizing hormone. Proc Natl Acad Sci USA 87:5163-5167.

Legan SJ, Tsai HW (2003) Oestrogen receptor- $\alpha$ and $\beta$-immunoreactivity in gonadotropin-releasing hormone neurones after ovariectomy and chronic exposure to oestradiol. J Neuroendocrinol 15:1164-1170.

Lloyd JM, Hoffman GE, Wise PM (1994) Decline in immediate early gene expression in gonadotropin-releasing hormone neurons during proestrus in regularly cycling, middle-aged rats. Endocrinology 134:1800-1805.

McCulloch DA, MacKenzie CJ, Johnson MS, Robertson DN, Holland PJ, Ronaldson E, Lutz EM, Mitchell R (2002) Additional signals from VPAC/PAC family receptors. Biochem Soc Trans 30:441-446.

Paxinos G, Watson C (1997) The rat brain in stereotaxic coordinates. New York: Academic.

Petersen SL, McCrone S, Keller M, Shores S (1995) Effects of estrogen and progesterone on luteinizing hormone-releasing hormone messenger ribonucleic acid levels: consideration of temporal and neuroanatomical variables. Endocrinology 136:364-410.

Roozendaal B, van Gool WA, Swaab DF, Hoogendijk JE, Mirmiran M (1987) Changes in vasopressin cells of the rat suprachiasmatic nucleus with aging. Brain Res 409:259-264.

Rubin BS, Lee CE, Ohtomo M, King JC (1997) Luteinizing hormonereleasing hormone gene expression differs in young and middle-aged females on the day of a steroid-induced LH surge. Brain Res 770:267-276.

Ruby NF, Edgar DM, Heller HC, Miller JD (1998) The aged suprachiasmatic nucleus is phase-shifted by cAMP in vitro. Brain Res 779:338-341.

Satinoff E, Li H, Tcheng TK, Liu C, McArthur AJ, Medanic M, Gillette MU (1993) Do the suprachiasmatic nuclei oscillate in old rats as they do in young ones? Am J Physiol 265:R1216-R1222.

Shinohara K, Tominaga K, Isobe Y, Inouye S-IT (1993) Photic regulation of peptides located in the ventrolateral subdivision of the suprachiasmatic nucleus of the rat: daily variations of vasoactive intestinal polypeptide, gastrin-releasing peptide, and neuropeptides Y. J Neurosci 13:793-800.

Simerly RB (1998) Organization and regulation of sexually dimorphic neuroendocrine pathways. Behav Brain Res 92:195-203.
Simerly RB, Chang C, Muramatsu M, Swanson LW (1990) Distribution of androgen and estrogen receptor mRNA-containing cells in the rat brain: an in situ hybridization study. J Comp Neurol 294:76-95.

Simonian SX, Spratt DP, Herbison AE (1999) Identification and characterization of estrogen receptor $\alpha$-containing neurons projecting to the vicinity of the gonadotropin-releasing hormone perikarya in the rostral preoptic area in the rat. J Comp Neurol 411:346-358.

Smith MJ, Jennes L, Wise PM (2000) Localization of the VIP2 receptor protein on GnRH neurons in the female rat. Endocrinology 141:4317-4320.

Stephan FS, Berkley KS, Moss RL (1981) Efferent connections of the rat suprachiasmatic nucleus. Neuroscience 6:2625-2641.

Swanson LW (1987) The hypothalamus. In: Handbook of chemical neuroanatomy, pp 1-124. Amsterdam: Elsevier.

Tanaka M, Ichitani Y, Okamura H, Tanaka Y, Ibata Y (1993) The direct retinal projection to VIP neuronal elements in the rat SCN. Brain Res Bull 31:637-640.

van der Beek EM, Horvath TL, Weigant VM, van den Hurk R, Buijs RM (1997) Evidence for a direct neuronal pathway from the suprachiasmatic nucleus to the gonadotropin-releasing hormone system: combined tracing and light and electron microscopic immunocytochemical studies. J Comp Neurol 384:569-579.

van der Beek EM, Swarts HJ, Wiegant VM (1999) Central administration of antiserum to vasoactive intestinal peptide delays and reduces luteinizing hormone and prolactin surges in ovariectomized, estrogen-treated rats. Neuroendocrinology 69:227-237.

Wise PM (1999) Neuroendocrine modulation of the "menopause": insights into the aging brain. Am J Physiol 277:E965-E970.

Wise PM (2000) New understanding of the complexity of the menopause and challenges for the future. In: Proceedings of the Serono conference on the biology of the menopause, pp 1-8. New York: Springer.

Wise PM, Smith MJ (2001) Neuroendocrine aspects of female reproductive aging. In: Functional neurobiology of aging (Hof PR, Mobbs CV, eds), pp 795-806. San Diego: Academic.

Wise PM, Camp-Grossman P, Barraclough CA (1981) Effects of estradiol and progesterone on plasma gonadotropins, prolactin, and LHRH in specific brain areas of ovariectomized rats. Biol Reprod 24:820-830.

Wise PM, Krajnak KM, Kashon ML (1996) Menopause: the aging of multiple pacemakers. Science 273:67-70.

Wise PM, Kashon ML, Krajnak KM, Rosewell KL, Cai A, Scarborough K, Harney JP, McShane T, Lloyd JM, Weiland NG (1997) Aging of the female reproductive system: a window into brain aging. Recent Prog Horm Res 52:279-303.

Wise PM, Smith MJ, Dubal DB, Wilson ME, Krajnak KM, Rosewell KL (1999) Neuroendocrine influences and repercussions of the menopause. Endocr Rev 20:243-248.

Yamazaki S, Straume M, Tei H, Sakaki Y, Menaker M, Block GD (2002) Effects of aging on central and peripheral mammalian clocks. Proc Natl Acad Sci USA 99:10801-10806. 